\title{
Time-Scale Detection of Microemboli in Flowing Blood with Doppler Ultrasound
}

\author{
Brian S. Krongold,* Akbar M. Sayeed, Mark A. Moehring, Member, IEEE, \\ James A. Ritcey, Merrill P. Spencer, and Douglas L. Jones
}

\begin{abstract}
Small formed elements and gas bubbles in flowing blood, called microemboli, can be detected using Doppler ultrasound. In this application, a pulsed constant-frequency ultrasound signal insonates a volume of blood in the middle cerebral artery, and microemboli moving through its sample volume produce a Doppler-shifted transient reflection.

Current detection methods include searching for these transients in a short-time Fourier transform (STFT) of the reflected signal. However, since the embolus transit time through the Doppler sample volume is inversely proportional to the embolus velocity (Doppler-shift frequency), a matched-filter detector should in principle use a wavelet transform, rather than a shorttime Fourier transform, for optimal results. Closer examination of the Doppler shift signals usually shows a chirping behavior apparently due to acceleration or deceleration of the emboli during their transit through the Doppler sample volume. These variations imply that a linear wavelet detector is not optimal.

We apply linear and quadratic time-frequency and time-scale detectors to a set of noise-corrupted embolus data. Our results show improvements of about $1 \mathrm{~dB}$ using the time-scale detectors versus an STFT-based detector signifying that embolus detection is best approached as a time-scale problem. A time-scale-chirp detector is also applied and is found to have the overall best performance by about $0.5-0.7 \mathrm{~dB}$ while coming fairly close (about $0.75 \mathrm{~dB})$ to a theoretical upper bound.
\end{abstract}

Index Terms-Doppler ultrasound, microemboli, time-frequency, time-scale, wavelet detection.

\section{INTRODUCTION}

T HE detection of small formed elements (such as blood clots) and gas bubbles in the bloodstream, known as microemboli, using pulse Doppler ultrasound is an important biomedical problem [1]-[4]. A small volume region in an artery is insonated using a constant-frequency ultrasound signal, and the reflections are sampled then processed in order to detect Doppler-shifted returns from these blood artifacts.

Manuscript received June 16, 1998; revised March 24, 1999. This work was supported by the Office of Naval Research under Contract N00014-951-0674. Asterisk indicates corresponding author.

*B. S. Krongold is with the Department of Electrical and Computer Engineering and Coordinated Science Laboratory, University of Illinois at Urbana-Champaign, Urbana, IL 61801 USA (e-mail: krongold@uiuc.edu).

A. M. Sayeed is with the Department of Electrical and Computer Engineering, University of Wisconsin-Madison, Madison, WI 53706 USA.

M. A. Moehring and M. P. Spencer are with Spencer Technologies, Inc., Seattle, WA 98122 USA.

J. A. Ritcey is with the Department of Electrical Engineering, University of Washington, Seattle, WA 98195-2500 USA.

D. L. Jones is with the Department of Electrical and Computer Engineering and Coordinated Science Laboratory, University of Illinois at Urbana-Champaign, Urbana, IL 61801 USA.

Publisher Item Identifier S 0018-9294(99)06802-0.
Without proper emboli detection, major medical complications can occur, including strokes and even death. Because they are highly nonstationary, embolus detection based on time-frequency representations (TFR's) have been considered. Current detection methods utilize the spectrogram, which is the squared magnitude of the short-time Fourier transform (STFT), or the Wigner distribution [5] to detect embolus transients in the reflected ultrasound signal [6]. This detector would potentially be optimal if the duration of the embolus signal remained constant with frequency (velocity). However, the transit time of the embolus through the Doppler sample volume is inversely proportional to its velocity, which suggests the use of a matched-filter detector using a wavelet transform.

The real part of four Doppler signals from emboli are shown in Fig. 1. The emboli in Fig. 1(a) and (b) clearly depict a scale (inverse relation between frequency and time-duration) relationship, which is precisely the expected physical relationship between emboli traveling at different velocities (with proportional Doppler shifts) through a fixed-length insonated section of an artery. Fig. 2(a), which plots the half-maximumamplitude time-durations of 95 emboli versus their center frequencies, also confirms the inverse relationship between inverse frequency (that is, scale) and duration in experimentally measured signals. This suggests that a time-scale-based, or wavelet-based, detector might yield an optimal matchedfilter detector for such signals [7], which motivates the study described in this paper. However, the scale relationship is not unitary because the amplitude of an embolus is not adjusted in accordance with its signal duration. Therefore, we expect higher total energy [and, thus, higher effective signal-to-noise ratios (SNR's)] in the longer-duration emboli than in the shorter ones, which complicates the detection problem slightly yet still suggests the use of a wavelet-based detector.

As will be discussed in Section II, the continuous wavelet transform is the ideal detector for constant-velocity (and, therefore, constant-frequency) emboli. However, many measured emboli signals exhibit varying frequency content, as can be observed in Fig. 1(c) and (d). These apparently reflect acceleration and deceleration of the emboli, perhaps due to the pulsatile nature of blood flow, interaction with the artery walls, or from turbulence or eddies in the flowing blood. Constant acceleration or deceleration causes a linear chirping effect [8] as clearly observed in Fig. 1(c). Fig. 2(b) plots the estimated chirp rate $^{1}$ of 95 measured emboli, confirming that this is

\footnotetext{
${ }^{1}$ The chirp rate was estimated using a least-squares linear fit of the embolus' instantaneous frequency.
} 


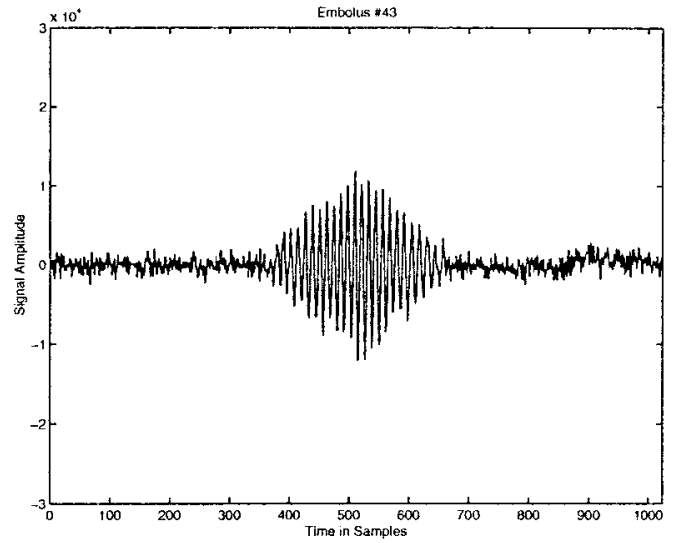

(a)

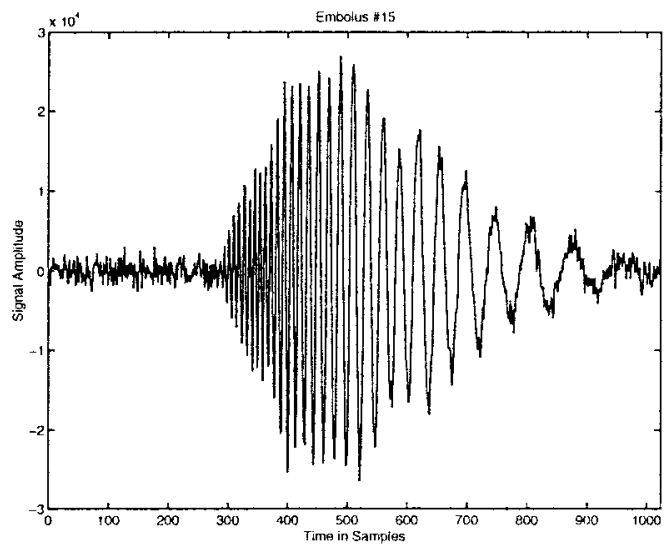

(c)

Fig. 1. Real part of typical emboli.

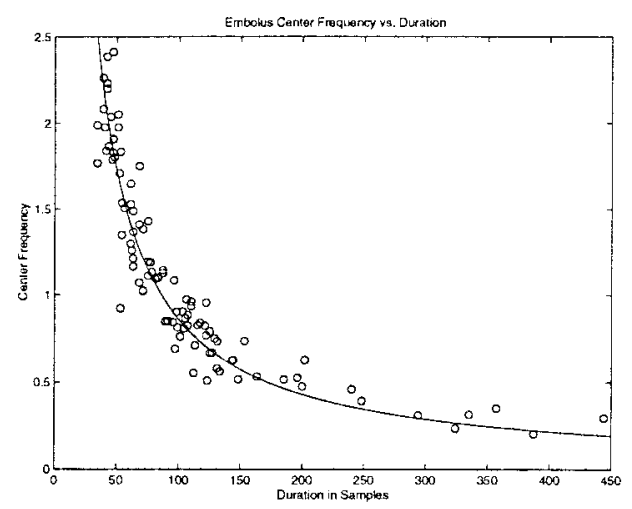

(a)

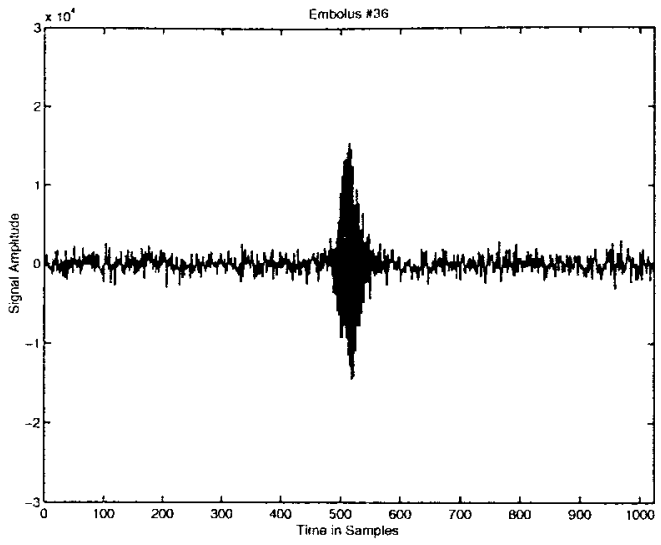

(b)

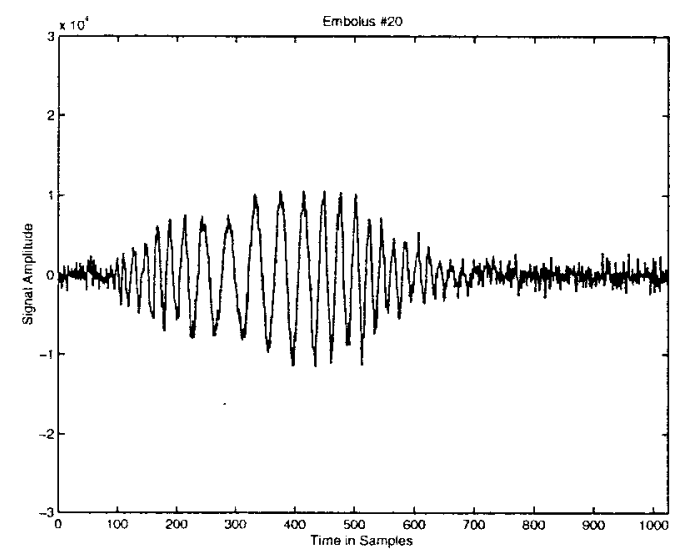

(d)

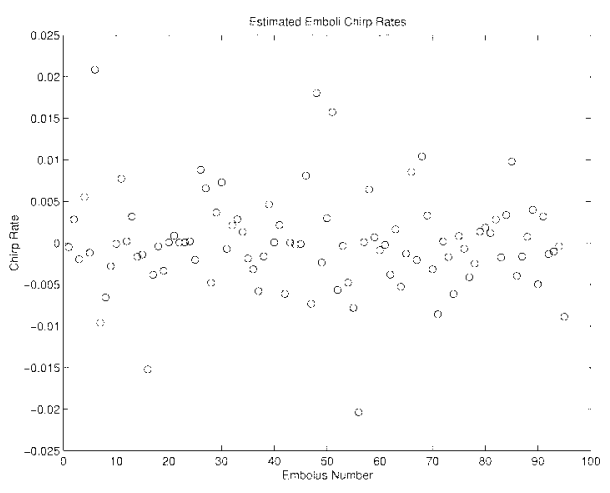

(b)

Fig. 2. Estimated emboli characteristics. (a) Center frequency versus duration. The solid curve is the best fit $C / t$ function where $C$ is a constant. (b) Chirp rate of each embolus.

a common phenomenon in real data. This effect alters the signal such that the constant-frequency wavelet is no longer an optimal matched filter for detection. For this reason, we introduce in this paper a linear chirp-wavelet (time-scalechirp) detector in the hopes of improving the detection of such emboli. However, we note in Fig. 1(d) that the frequency variation can be even more complicated (this embolus appears to undergo both deceleration and acceleration), thus rendering suboptimal the use of a time-scale-chirp detector. For this reason, we also investigate a quadratic time-scale detector as a means of dealing with variation in the embolus waveform.

In this paper, we employ time-frequency and time-scale detectors to detect these nonstationary emboli from reflected Doppler-ultrasound data. These include both linear and quadratic time-frequency and time-scale detectors, as well as a linear time-scale-chirp detector. The detection results are compared to a theoretically optimal "oracle" detector in which we know the exact form of each embolus prior to detection. In 
the next section, we provide a brief review of time-frequency and time-scale representations. In Section III, time-frequency and time-scale detection theories are reviewed, followed by a discussion of their implementation and formation of the necessary statistics in Section IV. Experimental results comparing the various detectors' performance for embolus detection are presented in Section V, and some conclusions are presented in the final section.

\section{TIME-FrEQUENCY/Time-ScALE REPRESENTATIONS}

TFR's are powerful methods for analyzing and processing nonstationary signals with time-varying spectral content. A one-dimensional signal, $s(t)$, is mapped by a TFR into a two-dimensional (2-D) signal, $T_{s}(t, f)$, which is a function of both time and frequency. This joint representation exploits the nonstationary characteristics of a signal and, therefore, can be very useful in detecting nonstationary signals.

The simplest and most popular TFR is the STFT, which is defined as [9]

$$
\mathrm{STFT}_{s}(t, f ; g) \equiv \int s(\tau) g^{*}(\tau-t) e^{-j 2 \pi f \tau} d \tau
$$

where $g(t)$ is called the analysis window. It can be interpreted as a local Fourier transform of $s(t)$ created by sliding the analysis window across the signal. The squared magnitude of the STFT is known as the spectrogram.

The continuous wavelet transform (CWT), the most popular time-scale representation (TSR), jointly represents a signal in terms of time and scale $(c>0)$, and is defined as [10]

$$
\mathrm{CWT}_{s}(t, c ; g) \equiv \frac{1}{\sqrt{c}} \int s(\tau) g^{*}\left(\frac{\tau-t}{c}\right) d \tau
$$

where $c$ is the scale at which the mother wavelet, $g(t)$, is projected onto the signal $s(t)$. Scale is a unitary operator which expands and compresses the duration of a signal while conserving signal energy. The squared magnitude of the CWT is known as the scalogram.

Bilinear or quadratic TFR's (Cohen's class) and TSR's (affine class) are more flexible than linear time-frequency representations and potentially offer improved detection performance. Both classes are defined as smoothed versions of the Wigner distribution (WD), which is defined as

$$
W_{s}(t, f) \equiv \int s\left(t+\frac{\tau}{2}\right) s^{*}\left(t-\frac{\tau}{2}\right) e^{-j 2 \pi f \tau} d \tau
$$

A quadratic TFR in Cohen's class, which is a generalization of the spectrogram, involves convolutional smoothing of the WD [8]

$$
P_{s}(t ; f ; \Phi) \equiv \iint W_{s}(u, v) \Phi(u-t, v-f) d u d v
$$

where the 2-D kernel $\Phi$ completely characterizes the TFR $P_{s}(\Phi)$. A quadratic TSR in the affine class is a generalization of the scalogram and is characterized by an affine smoothing of the WD [9]

$$
C_{s}(t ; c ; \Pi) \equiv \iint W_{s}(u, v) \Pi\left(\frac{u-t}{c}, c v\right) d u d v
$$

where the kernel $\Pi$ completely characterizes the TSR $C_{s}(\Pi)$.

Cohen's class and the affine class can be expressed as a weighted sum of spectrograms and scalograms, respectively, as [11]

$$
\begin{aligned}
& P_{s}(t ; f ; \Phi)=\sum_{k} \lambda_{k} \mid\left(\left.\operatorname{STFT}_{s}\left(t, f ; u_{k}\right)\right|^{2}\right. \\
& C_{s}(t ; f ; \Pi)=\sum_{k} \mu_{k} \mid\left(\left.\mathrm{CWT}_{s}\left(t, f ; v_{k}\right)\right|^{2}\right.
\end{aligned}
$$

where the $\lambda_{k}$ 's and $u_{k}$ 's represent the eigenvalues and orthonormal eigenvectors, respectively, of the linear operator defined by the kernel $\Phi$. This same relationship is true for the $\mu_{k}$ 's and $v_{k}$ 's with the kernel $\Pi$.

\section{TIME-FREQUENCY/TIME-SCALE DETECTION}

We will consider the following hypothesis testing problem of the form

$$
\begin{aligned}
& H_{1}: y(t)=s(t ; \tau, f \text { or } c)+n(t) \\
& H_{0}: y(t)=n(t)
\end{aligned}
$$

where $t \in T$ is the observation interval, $y$ is the observed signal, $s$ is the underlying signal to be detected, and $n$ is additive noise. In the embolus detection problem, the hypothesis $H_{0}$ represents the case that no embolus is present, while $H_{1}$ is the situation in which an embolus is present along with the background electronic or bloodflow noise. Two different classes of detectors, linear and quadratic, will be discussed in the remainder of this section.

\section{A. Linear TF/TS Detection}

The underlying assumption for using linear detectors is that the signal to be detected is known and deterministic. In this case, it is well known that, in white Gaussian noise, the matched filter is the optimal detector [12] and corresponds to the test statistic

$$
L(y)=\langle y(t), s(t)\rangle
$$

In certain situations, the received signal may undergo unknown time, frequency, and/or scale shifts. This is known as a composite hypothesis test, in which the goal is to detect $s(t ; \tau, f$ or $c)$ with unknown parameters. A uniformly most powerful rule [12] does not exist in this case, and in practice a generalized likelihood-ratio test (GLRT) is usually adopted. The GLRT is defined as follows, with the linear detector form explicitly shown:

$$
\begin{aligned}
L_{\mathrm{GLRT}}(y) & =\max _{\tau, f \text { or } c} L^{(\tau, f \text { or } c)}(y) \\
& =\max _{\tau, f \text { or } c}\langle y(t), s(t ; \tau, f \text { or } c)\rangle .
\end{aligned}
$$


We note that in the special case where the deterministic waveform to be detected undergoes unknown time and frequency shifts, the required inner products at the various time and frequency offsets are exactly the outputs of a short-time Fourier transform using the matched filter as the window! Similarly, for unknown time and scale shifts, the necessary inner products are values of the continuous wavelet transform adopting the matched filter as the wavelet. This equivalence forms the fundamental theoretical basis for optimal time-frequency and time-scale (wavelet-based) detection, as well as offering a means for their efficient implementation. As argued in the Introduction, the physical process creating Doppler-ultrasound embolus signals suggests wavelet-based (time-scale) detectors are ideally matched to this detection problem.

A chirp parameter can be also be introduced by adding a third dimension into the GLRT search for the time-scale detector. This chirp parameter modifies the underlying signal in the matched filter by

$$
s(t ; \tau, c, \theta)=s(t ; \tau, c) e^{j \theta t^{2}}
$$

where $\theta$ is the newly introduced chirp-rate parameter. This linear time-scale-chirp detector can be defined as follows:

$$
L_{\mathrm{GLRT}}(y)=\max _{\tau, c, \theta} L^{(\tau, c, \theta)}(y)=\max _{\tau, c, \theta}\langle y(t), s(t ; \tau, c, \theta)\rangle .
$$

As mentioned in the Introduction, the acceleration or deceleration of emboli through the Doppler transit volume causes a chirping in the received ultrasound signal, thereby suggesting the use of a chirp parameter in the GLRT detector.

\section{B. Quadratic TF/TS Detection}

A glance at the signals from the emboli in Fig. 1 is sufficient to confirm that, even after time and frequency or scale changes, these signals are not exactly identical. The linear detectors are known to be optimal only for detecting deterministic signals in Gaussian noise and, thus, a more sophisticated detector may be required to obtain optimal performance in this problem. We will now consider the following hypothesis testing problem [7]:

$$
\begin{aligned}
H_{1} & : y(t)=s(t ; \tau, c)+n(t) \\
H_{0} & : y(t)=n(t) \\
y(t) & : \text { Observed signal; } \\
s(t ; \tau, c) & : \text { Zero-mean Gaussian signal; } \\
(\tau, c) & : \text { Known time and scale parameters; } \\
n(t) & : \text { White Gaussian noise. }
\end{aligned}
$$

The key difference between this and the linear hypothesis test is that the signals to be detected are no longer deterministic, but are random Gaussian signals with a known autocorrelation structure. This model provides a mechanism for capturing some of the variations in the signals from different emboli. We will also define $s_{0}$ with autocorrelation function $\mathbf{R}_{0}$ as our nominal signal. Therefore, every $s(t ; \tau, c)$ is just a scaled and time-shifted version of $s_{0}$, and since scale and time shifts are just linear operators, the autocorrelation function of $s(t ; \tau, c)$ is just a scaled and shifted version of $\mathbf{R}_{0}$. This is shown in the following statistical summary of the above hypothesis-testing scenario:

$$
\begin{aligned}
s(t ; \tau, c) & =\frac{1}{\sqrt{c}} s_{0}\left(\frac{t-\tau}{c}\right) \\
s_{0}(t) & \sim \mathcal{N}\left(0, \mathbf{R}_{0}\right) \\
\mathbf{R}_{s}^{(\tau, c)}\left(t_{1}, t_{2}\right) & =\frac{1}{c} \mathbf{R}_{0}\left(\frac{t_{1}-\tau}{c}, \frac{t_{2}-\tau}{c}\right) \\
n(t) & \sim \mathcal{N}\left(0, \sigma^{2} \mathbf{I}\right)
\end{aligned}
$$

where $\mathcal{N}$ represents a Gaussian distribution, $\mathbf{I}$ is the identity matrix, and $\sigma^{2}$ is the noise variance.

We will briefly assume that scale and time shifts are not present. In this case, classical detection theory then tells us that the optimal detector compares a quadratic function of the observations to a threshold to decide whether $H_{1}$ or $H_{0}$ is true. This optimal test statistic can be written in the form [12]

$$
\begin{aligned}
L(y) & =\langle\mathbf{Q} y, y\rangle \\
Q\left(t_{1}, t_{2}\right) & =\sum_{k} \frac{\lambda_{k}}{\lambda_{k}+\sigma^{2}} v_{k}\left(t_{1}\right) v_{k}^{*}\left(t_{2}\right)
\end{aligned}
$$

where $\mathbf{Q}$ is a positive-definite linear operator with the $v_{k}$ 's and $\lambda_{k}$ 's as the eigenvectors and corresponding eigenvalues of $\mathbf{R}_{0}$.

Referring back to the case of known scale and time shifts, $Q$ is dependent upon $(\tau, c)$, since the autocorrelation function, $\mathbf{R}_{s}^{(\tau, c)}$, changes with scale and time shifts. Therefore, we must obtain an eigenexpansion of the autocorrelation function in order to obtain $Q^{(\tau, c)}$ in the form of (16).

Since scaling and time shifting are unitary operators, the eigenvalues of $\mathbf{R}_{s}^{(\tau, c)}$ are the same as those of $\mathbf{R}_{0}$, whereas the eigenvectors are simply time-shifted and scaled versions of the eigenvectors of $\mathbf{R}_{0}$. Therefore, we can now write (16) as

$$
Q^{(\tau, c)}\left(t_{1}, t_{2}\right)=\sum_{k} \frac{\lambda_{k}}{\lambda_{k}+\sigma^{2}} \frac{1}{\sqrt{c}} v_{k}\left(\frac{t_{1}-\tau}{c}\right) \frac{1}{\sqrt{c}} v_{k}^{*}\left(\frac{t_{2}-\tau}{c}\right) .
$$

Substituting this result into (15), bringing the integrals inside the summation, and using the definition of the CWT in (2) we obtain

$$
\begin{aligned}
L^{(\tau, c)}(y) & =\sum_{k} \alpha_{k}\left|\mathrm{CWT}_{y}\left(\tau, c ; v_{k}\right)\right|^{2} \\
\text { where } \alpha_{k} & =\frac{\lambda_{k}}{\lambda_{k}+\sigma^{2}} .
\end{aligned}
$$

We now have a relationship for the optimal test statistic for any $(\tau, c)$ in terms of magnitude-squared CWT's, also known as scalograms. In addition, $L^{(\tau, c)}(y)$ is a TSR, since it is the weighted sum of scalograms. Therefore, every point in the TSR is the optimal test statistic for the corresponding time-shift and scale.

When $(\tau, c)$ are unknown parameters, the hypothesis testing scenario of (13) becomes a composite hypothesis test with parameters $(\tau \in \Re, c>0)$. A uniformly most powerful rule does not exist in this case and the GLRT which corresponds 
to the maximum-likelihood (ML) estimate of $(\tau, c)$ is used. This is expressed as [7]

$$
L_{\mathrm{GLRT}}(y)=\max _{\tau, c} L^{(\tau, c)}(y)
$$

Using the bank of scalograms as in (18) and taking the maximum value, we can obtain this statistic and implement the GLRT via wavelet transforms.

The time-scale detector corresponds to a quadratic timescale representation with a kernel related to $Q^{(\tau, c)}$ in (16). Quadratic time-frequency detectors can be derived in a similar manner, with frequency replacing scale and short-time Fourier transforms replacing continuous wavelet transforms in the above analysis. These equivalences lead to a variety of implementation alternatives and additional insights; while we discuss here only those aspects most relevant to the problem of ultrasound embolus detection, a much more complete development of quadratic time-frequency and time-scale detectors can be found in [7].

\section{DETECTOR Formation AND IMPLEMENTATION}

In this section, we outline the formation and implementation details of the GLRT detectors used for our studies. As in the previous section, the linear detectors are discussed first, followed by the quadratic ones. Three different linear detectors are considered: time-scale (CWT), time-frequency (STFT), and time-scale-chirp (Chirp-CWT). Time-scale (TS) and timefrequency (TF) detectors are considered in the quadratic case.

\section{A. Linear Detectors}

The implementation of the linear detectors is relatively straightforward. An appropriate wavelet/window is selected for the CWT and STFT via experimentation, and the GLRT maximum magnitude search consists only of computing the magnitude of the corresponding CWT or STFT, determining the maximum over the range of scales or frequencies at which emboli can appear, and comparing this maximum to a threshold. We selected a truncated bandpass Gaussian wavelet for the CWT, and the STFT was computed using a length128 Hamming window. ${ }^{2}$ It should be noted that the embolus signal envelope and, therefore, the optimal choice of wavelet and STFT window, is dependent upon the Doppler sample volume which will change with the depth of the vessel.

An equivalent number of detector points were computed in the CWT and the STFT. The CWT used 128 scales and one-sample time shifts, whereas the STFT computed 512 frequencies with window shifts of four samples. The CWT was computed efficiently utilizing the chirp-z transform and fast convolution [13]. The time-scale-chirp detector used the same CWT for nine different chirp rates, and the GLRT search was done over this parameter space. The search over frequency/scale in the GLRT was done only for a range bounded by the center frequencies/scales of the measured emboli.

\footnotetext{
${ }^{2}$ Although different windows were chosen for the two detectors, Gaussian and Hamming windows (of optimized duration) gave virtually identical performance for each. These windows were chosen to reflect existing practice.
}

\section{B. Quadratic TF/TS Detectors}

Implementation of the quadratic TF/TS detectors requires a statistical model of the emboli centered at some nominal time and frequency/scale. The DC components of the emboli were extremely small anyway, and were simply removed to fit the zero-mean Gaussian model in (13). The autocorrelation function, $\mathbf{R}_{0}$, at some nominal TF/TS point was required in order to obtain the eigenvalues and eigenvectors used in the detector. This is not straightforward, since all of our signals are centered at various frequencies/scales and have different time shifts. Therefore, the training signals must be aligned by modulating/scaling and time-shifting the data to the same nominal point in the TF/TS plane. This aligned data was then used to form $\mathbf{R}_{0}$. As in the linear detector case, $\mathbf{R}_{0}$ is also dependent on the Doppler sample volume and the vessel depth.

For alignment, a reference emboli, shown in Fig. 2(a), was selected, and the signal portion of this realization was extracted to serve as a "template." This template was correlated with modulated/scaled and time-shifted versions of the other emboli, the scale/frequency and time shifts yielding the maximum correlation with the template were determined, and the other emboli signals were shifted in the TF/TS plane to be aligned with the template. This procedure is intuitively appealing, although many of the emboli do not have constant frequency/scale over the entire signal, and the alignment is occasionally not visually pleasing. Theoretical examples can be constructed which suggest that this alignment procedure is not optimal; nonetheless, it has yielded good results in practice.

After obtaining a set of aligned data, we compute the correlation statistics by computing outer products of the vectors, adding up the results, and dividing by the number of realizations. In theory, this is asymptotically optimal, but a slight complication arises here due to a consistent variation in energy across scales. Our model assumes that scaling is a unitary (and, therefore, energy-conserving) operation. However, in Doppler ultrasound measurement of microemboli, the durations vary with velocity (and, thus, scale or frequency), but the amplitudes are not affected by their velocity. This poses a problem when trying to form $\mathbf{R}_{0}$ from the experimental data without letting lower frequency or large-amplitude emboli dominate the statistics. The solution we chose was to energy-normalize the aligned data vectors before estimating the correlation matrix, $\mathbf{R}_{0}$, thus, giving each embolus an equal contribution to $\mathbf{R}_{0}$.

Plots of the eigenvalues ${ }^{3}$ of the $\mathbf{R}_{0}$ for the quadratic $\mathrm{TF}$ and TS detectors are shown in Fig. 3. The first eigenvalue dominates in both the TF and TS case, and the overall eigenvalue spread is better for the TS detector than the TF detector $(93.6 \%$ of the eigenvalue sum is contained in the first ten eigenvalues of the TS detector while the TF detector contains only $80.9 \%$ in its first ten). Since most of the eigenvalues are very small, it is pointless to include their weight in the test statistic in (17). Thus, a reducedrank detector utilizing only the significant eigenvalues can be implemented, and the overall cost of the quadratic detectors will be approximately $N$ times that of a linear detector, where

\footnotetext{
${ }^{3} \mathbf{R}_{0}$ is normalized so that the sum of its eigenvalues is equal to one.
} 


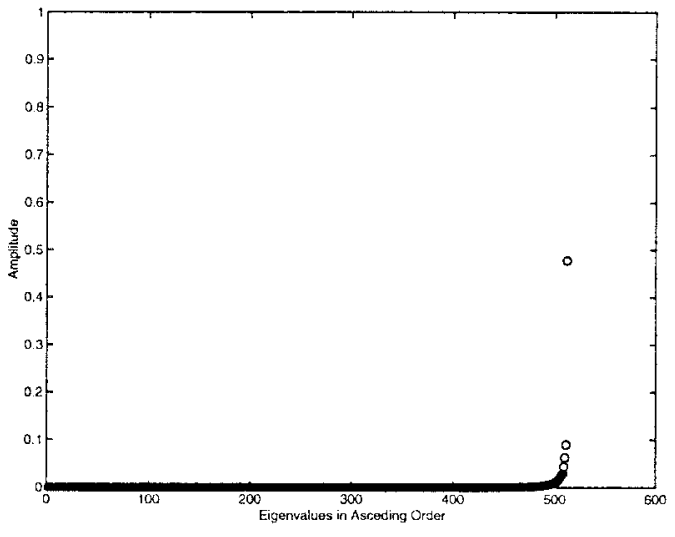

(a)

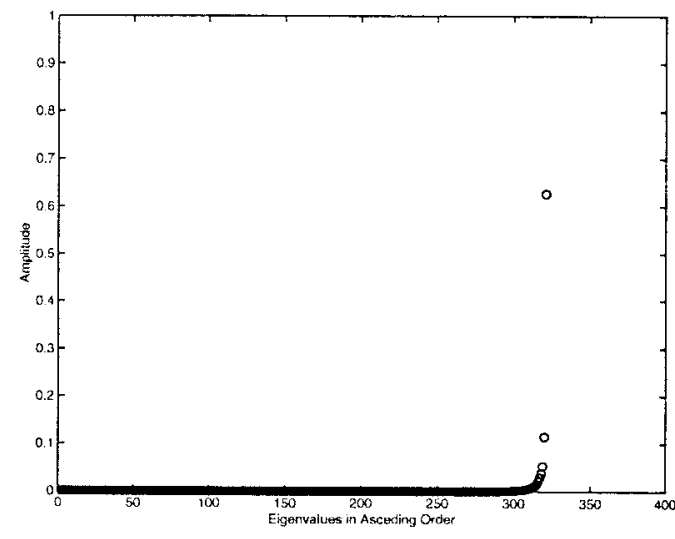

(b)

Fig. 3. Eigenvalues of both quadratic detectors. (a) Time-frequency. (b) Time-scale.

$N$ is the chosen rank. The individual scalograms are simply the squared magnitude of continuous wavelet transforms (or STFT's in the quadratic time-frequency detector) using the eigenvectors as the wavelets (or windows).

\section{RESULTS}

A patient with a mechanical aortic valve, who was previously diagnosed to have middle cerebral artery (MCA) embolic signals that could be traced by both common carotid arteries to the heart, was restudied with a novel pulse Doppler. The Doppler pulse repetition frequency was $8 \mathrm{kHz}$, and each transmit burst was comprised of 24 cycles at $2.4 \mathrm{MHz}$. The sample gate depth was $5 \mathrm{~cm}$. Signals were acquired through the temporal bone using a wide-beam probe (7- $\mathrm{mm}$ diameter). A customized headgear was positioned on the patient so the probe could be trained on the MCA and held in position for several hours at a time. Embolic signals were gathered, and the time-series Doppler shift signal within a two-second window surrounding it was digitized and saved to disk.

The SNR of the raw measurements is relatively high (about $30 \mathrm{~dB}$ ), allowing easy visual identification and isolation of the embolus signals. For this experiment, 95 length-1024 records, each containing a signal from a single passing embolus (see Fig. 1) were extracted from the time-series ultrasound data.

The performance in more substantial noise was investigated by adding white Gaussian noise at various SNR levels (ten realizations per embolus). Since the energy of each embolus varied, SNR was defined to be the ratio of the average peak magnitude squared to the noise variance. A constant false alarm rate (CFAR) of 0.01 was set over a block of 1024 samples, thereby establishing a threshold to determine the number of emboli detected. While this false alarm rate is unrealistically high for a real application, it allowed accurate relative comparisons of performance with a modest number of trials; we expect that the relative performance of the various detectors will remain consistent at realistic CFAR levels. White noise appears to be a fairly accurate model of both electronics noise and the background reflections from individual corpuscles and other blood components (at least up to the maximum velocity), so we believe that these results are indicative of the performance in real low-SNR environments.

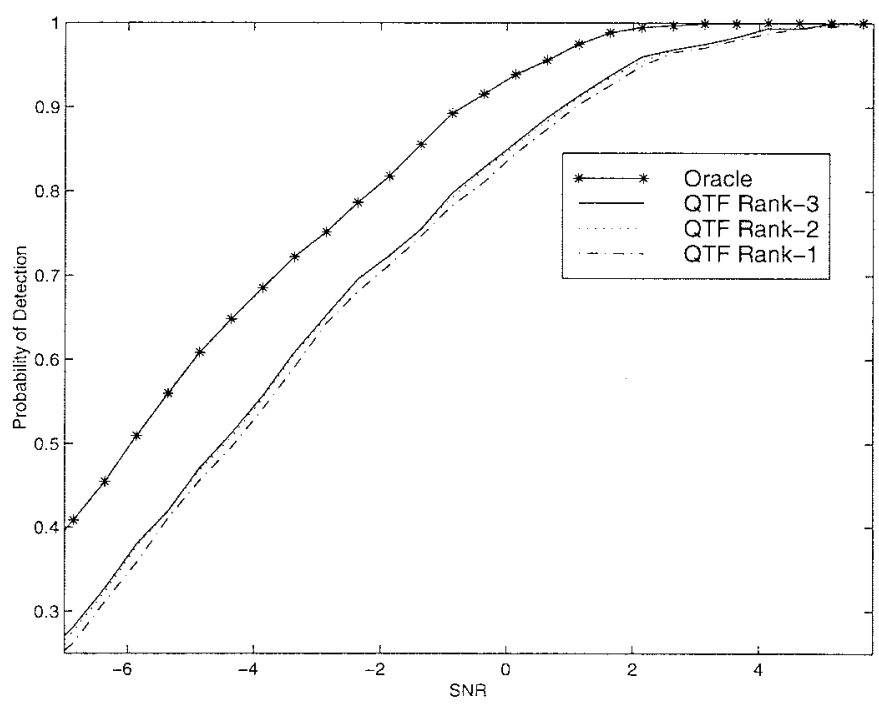

Fig. 4. Probability of detection results for quadratic TF detectors: $P_{f}=.01$.

Probability of detection results can be compared to that of an "oracle detector." By oracle detector, we refer to a detector which assumes exact, a priori knowledge of the individual embolus signals and, thus, applies the perfect matched filter for detection. Hence, the oracle detector serves as an unrealizable theoretical upper bound on the performance of the various detectors. In order to remain consistent with the other detectors in terms of the number of detector points and the CFAR threshold, the oracle detector was implemented using the timescale detector with the a priori known embolus used as the wavelet for each of the 95 emboli.

Fig. 4 shows the detection results for quadratic TF detectors of rank one through three. We can easily see the performance gain of a higher ranked detector; however, the gain is only about $0.25 \mathrm{~dB}$ for the rank-3 over the rank- 1 detector. This is as expected, since the first eigenvalue of the aligned correlation matrix dominates the rest as shown in Fig. 3(a). The same phenomenon occurs with the quadratic TS detector results shown in Fig. 5. We observe an even smaller performance gain from rank-1 to rank-3 in this case (about $0.15-0.25 \mathrm{~dB}$ ) than the TF case. Since the first eigenvalue dominates more 


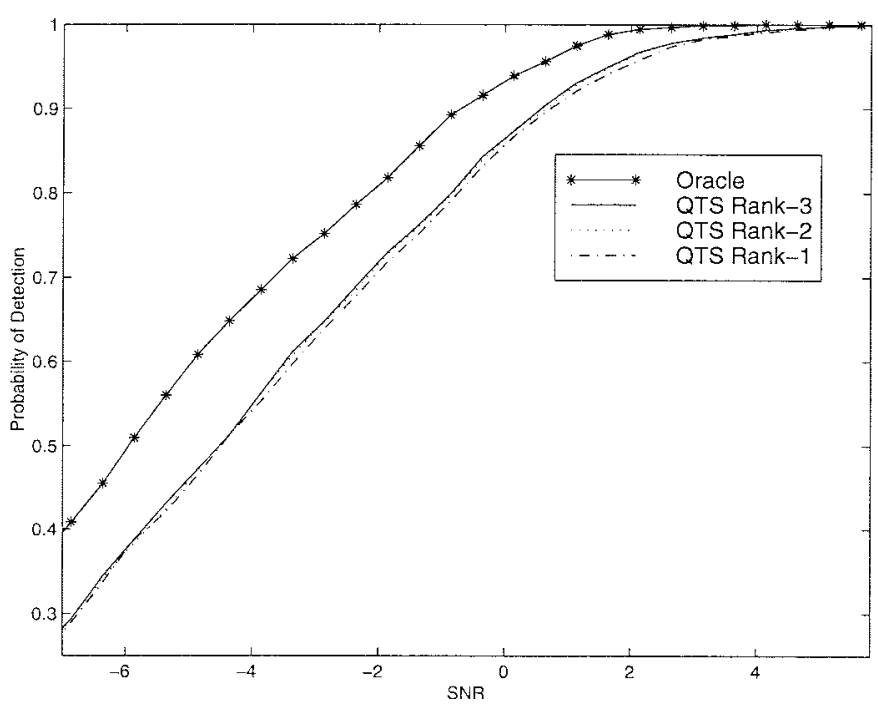

Fig. 5. Probability of detection results for quadratic TS detectors: $P_{f}=.01$.

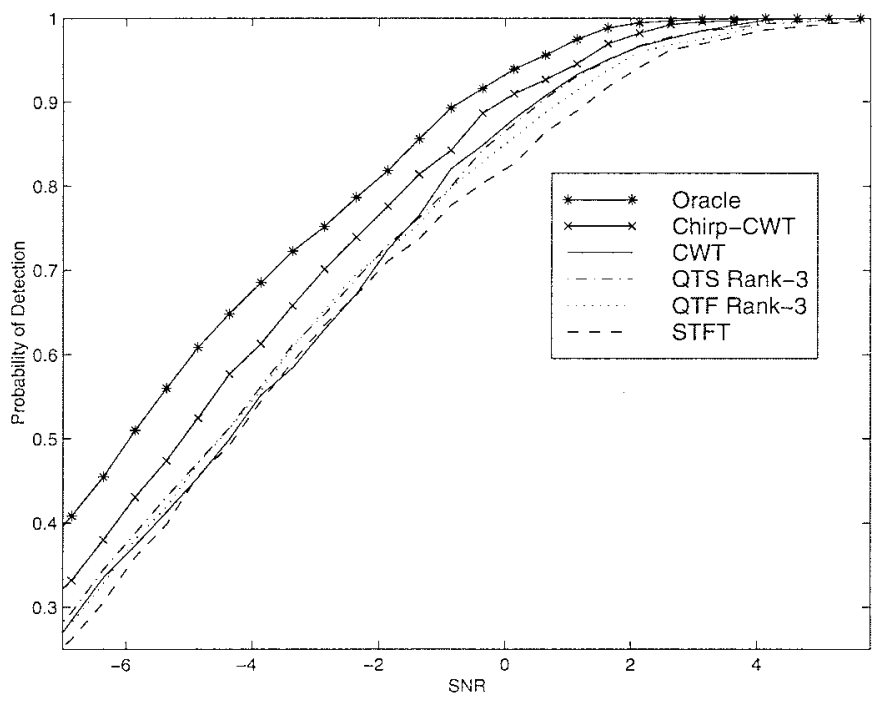

Fig. 6. Probability of detection comparison of linear and quadratic detectors: $P_{f}=.01$.

in the TS case [see Fig. 3(b)] than in the TF case, this result is also expected. The rank-3 quadratic TS detector is about $1.25-1.5 \mathrm{~dB}$ from the oracle bound while the rank-3 TF is about $1.5-1.75 \mathrm{~dB}$ away.

Fig. 6 is an overall comparison of linear and quadratic TF and TS detectors. We see that the time-scale methods easily outperform the STFT detector at high SNR's. We estimate a performance gain of about $1 \mathrm{~dB}$ for the CWT over the STFTbased method. The more expensive chirp-CWT detector is about $0.5-0.7 \mathrm{~dB}$ better than the CWT and only about $0.75 \mathrm{~dB}$ from the oracle bound. It should be noted that in addition to having at least the same or higher detector output as the CWT, the chirp CWT will also have an equal or greater noise output level as well, thus, raising the detection threshold for the CFAR detector. It appears that chirp variations are indeed a problem for the CWT detector by itself, and that chirp is a significant detection parameter.

The linear STFT (the current state-of-the-art) is indeed the poorest performer of the chosen methods. The rank-3 quadratic
TF detector has about a $0.5-\mathrm{dB}$ performance gain over the STFT, showing that variations among emboli are important when using the time-frequency model. However, linear and quadratic TS detectors perform better than the quadratic TF detector, thus confirming the notion that microemboli detection is best approached as a time-scale problem.

Another important observation (one which was unexpected at first) is that the linear CWT detector very slightly outperforms the rank-3 quadratic TS detector in some regions. This can be reasoned by noting that the linear CWT detector is experimentally optimized to perform well in terms of detection probability for the embolus set. It takes into account that certain emboli are harder to detect than other emboli due to varying signal energies. The quadratic TS detector is based upon a statistical formulation of the 95 emboli with each having an equal contribution to the resulting detector. It, therefore, does not take advantage of certain emboli being easier to detect than others, leaving room for the linear detector to have a performance advantage. On the other hand, by providing a robustness to variations in emboli, the quadratic detector is able to have nearly the same performance. Apparently, accounting for these variations may not be as important as compensating for emboli which are harder to detect. The fact that the CWT's performance relative to the quadratic TS detector is better at high detection probabilities, but worse at lower detection probabilities, also supports this hypothesis. We could have easily weighted the harder-to-detect emboli (lower energy) in the quadratic detector formation to achieve better results; however, this would be statistically biased and the results might be suspect. Furthermore, it is possible that the nonideal alignment for quadratic detector formation as well as insufficient amount of training data may also be a cause of this slight performance difference.

\section{CONCLUSION}

This paper has reviewed the theory of linear and quadratic time-frequency and time-scale detectors and performed a relatively thorough case study of their application to the Doppler ultrasound detection of microemboli. The use of timefrequency or time-scale detectors in practice should ideally be motivated by solid justification based on physical principles related to a given detection problem. In the case of embolus detection, the inverse relationship between Doppler frequency and time-duration, which is exactly equivalent to that in timescale transformations, strongly suggests that the continuous wavelet transform or quadratic time-scale detectors are ideal. The chirping behavior due to acceleration or deceleration of an embolus through the Doppler sample volume further suggests the use of a chirp parameter in the time-scale detector. The comparison of the relative performance of various detectors presented here supports these assessments. The CWT and the quadratic time-scale detectors yield equivalent detection performance to the currently-used STFT at about a 1-dB lower SNR, and the chirp-CWT detector outperformed the time-scale detectors by about $0.5-0.7 \mathrm{~dB}$.

In determining the preferred detector for a practical system, complexity, cost, and convenience issues must be weighed 
against the relative detection performance. The use of fast algorithms for computing the CWT allows the computational efficiency to approach that of the STFT for densely sampled transforms. However, considerable savings in computation can be obtained with the STFT when the time-step in samples between successive FFT's is greater than one, whereas the known CWT algorithms cannot efficiently exploit this reduction. In practice, this might mean that implementation of the CWT could require as much as ten times more computation than the STFT, which must be weighed against the improved detector performance. The quadratic TSR can require several CWT computations while offering similar performance as a single CWT, suggesting that they it not be worth the added complexity in this particular application. The chirp-CWT detector requires multiple CWT computations to obtain its demonstrated slightly higher performance, thus costing a few times more than the CWT detector. This extra implementation cost must be weighed against the performance gain to determine its suitability in practice.

Comparison with an oracle detector revealed that all of the methods studied here, including the relatively straightforward STFT-based detectors, come within about $0.75-2.25 \mathrm{~dB}$ of a theoretically unrealizable upper bound. In short, all of these methods perform quite well, and for this application no other non-TF/TS-based detection method could hope to achieve more than modest improvements over the methods presented here.

\section{REFERENCES}

[1] M. A. Moehring and J. A. Ritcey, "Sizing emboli in blood using pulse Doppler ultrasound-I: Verification of the EBR model," IEEE Trans. Biomed. Eng., vol. 43, pp. 572-580, June 1996.

[2] M. A. Moehring, J. A. Ritcey, and A. Ishimaru, "Sizing emboli in blood using pulse Doppler ultrasound-II: Effects of beam refraction," IEEE Trans. Biomed. Eng., vol. 43, pp. 581-588, June 1996.

[3] M. A. Moehring and J. R. Klepper, "Pulse Doppler ultrasound detection, characterization and size estimation of emboli in flowing blood," IEEE Trans. Biomed. Eng., vol. 41, pp. 35-44, Jan. 1994.

[4] M. P. Spencer, G. I. Thomas, S. C. Nicholls, and L. R. Sauvage, "Detection of middle cerebral artery emboli during carotid endarterectomy using transcranial Doppler ultrasonography," Stroke, vol. 21, pp. 415-423, Mar. 1990.

[5] J. L. Smith, D. H. Evans, L. Fan, A. J. Thrush, and A. R. Naylor, "Processing Doppler ultrasound signals from blood-borne emboli," Ultrasound Med. Biol., vol. 20, no. 5, pp. 455-462, 1994.

[6] R. E. Hileman, M. P. Spencer, and J. M. Reid, "Ultrasound physical concepts," in Ultrasound Diagnosis of Cerebrovascular Disease, M. P. Spencer, Ed. Dordrecht, the Netherlands: Martinus Nijhoff, 1987, pp. $7-28$.

[7] A. M. Sayeed and D. L. Jones, "Optimal detection using bilinear time-frequency and time-scale representation," IEEE Trans. Signal Processing, vol. 43, pp. 2872-2883, Dec. 1995.

[8] L. Cohen, Time-Frequency Analysis. Englewood Cliffs, NJ: PrenticeHall, 1995.

[9] F. Hlawatsch and G. F. Boudreaux-Bartels, "Linear and quadratic timefrequency signal representations," IEEE Signal Processing Mag., Apr. 1992.

[10] M. Vetterli and J. Kovacevic, Wavelets and Subband Coding. Englewood Cliffs, NJ: Prentice-Hall, 1995.

[11] R. G. Shenoy and T. W. Parks, "The Weyl correspondence and timefrequency analysis," IEEE Trans. Signal Processing, vol. 42, pp. 318-332, Feb. 1994

[12] H. V. Poor, An Introduction to Signal Detection and Estimation, 2nd ed. New York: Springer-Verlag, 1994

[13] D. L. Jones and R. G. Baraniuk, "Efficient approximation of continuous wavelet transforms," Electron. Lett., vol. 27, no. 9, pp. 748-750, Apr. 1991.

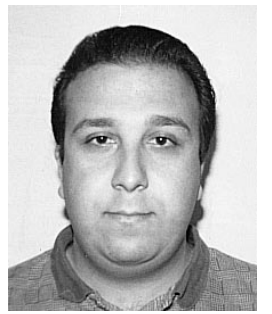

Brian S. Krongold received the B.S. and M.S. degrees in electrical engineering in 1995 and 1997, respectively, from the University of Illinois at UrbanaChampaign, where he is currently working toward the Ph.D. degree as a Research Assistant at the Coordinated Science Laboratory.

During summer 1994, he interned for Martin Marietta at the Oak Ridge National Laboratory, Oak Ridge, TN. From January to August of 1995, he consulted at Bell Laboratories in Middletown, NJ. During summer 1998, he worked at the Electronics and Telecommunications Research Institute, Taejon, South Korea under a National Science Foundation summer research fellowship. His research interests are in multicarrier communication systems, time-frequency/time-scale analysis and detection, and wavelets and filterbanks.

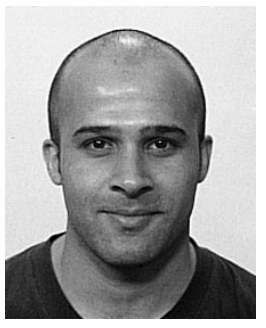

Akbar M. Sayeed received the B.S. degree from the University of Wisconsin, Madison, in 1991, and the M.S. and Ph.D. degrees in 1993 and 1996, respectively, from the University of Illinois at UrbanaChampaign, all in electrical engineering.

While at the University of Illinois, he was a Research Assistant in the Coordinated Science Laboratory and was also the Schlumberger Fellow in signal processing from 1992-1995. During 1996-1997, he was a Post-doctoral Fellow at Rice University, Houston, TX. Since August 1997, he has been with the University of Wisconsin-Madison, where he is currently an Assistant Professor. His research interests are in signal processing for wireless communications, statistical and time-varying signal processing, and time-frequency and wavelet analysis.

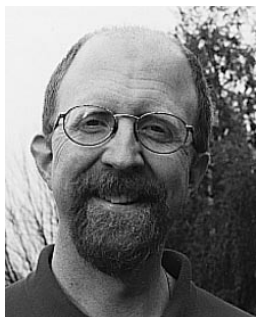

Mark A. Moehring (M'87) was born in Seattle, WA, in 1960. He received the B.S. degree in physics from Harvey Mudd College, Claremont, CA, in 1983, and the M.S.E.E. and Ph.D. degrees from the University of Washington, Seattle, in 1989 and 1994, respectively.

He was with the Institute of Applied Physiology and Medicine from 1983 to 1995, and since 1995 has held the positions of Senior Scientist and Director of Research and Development at Spencer Technologies, Seattle, WA. His research interests include biomedical applications of stochastic signal processing, with particular emphasis in physiological measurements using ultrasound.

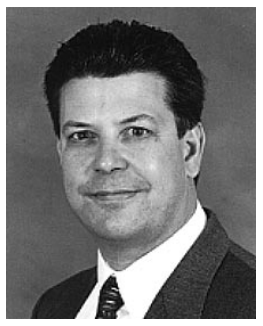

James A. Ritcey received the B.S.E. degree from Duke University, Durham, NC. in 1976, the M.S.E.E. degree from Syracuse University, Syracuse, NY, in 1980, and the Ph.D. degree in electrical engineering (communication theory and systems) from the University of California, San Diego (UCSD) in 1985.

Since 1985, he has been with the Department of Electrical Engineering at the University of Washington, where he now holds the rank of Professor. From 1981 to 1985 , he was a graduate Research Assistant at the Department of Electrical Engineering and Computer Sciences at UCSD. From 1976 to 1981 he was with the General Electric Company, and graduated from GE's Advanced Course in Engineering. His research interests include communications and statistical signal processing for radar, sonar, and biomedicine.

Professor Ritcey has served as Technical Program Chair of the 1992 Asilomar and General Chair of the 1994 Asilomar Conference on Signals, Systems, and Computers. He served as the General Chair of the 1995 International Conference on Communications. 


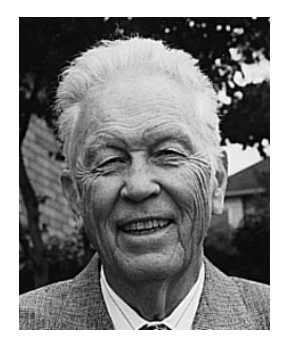

Merrill P. Spencer was born in Pawnee, OK, in 1922. He received the B.S. degree from Oklahoma Baptist University, Shawnee, and the M.D. degree in 1945 from Baylor University College of Medicine, Waco, TX.

He has held the positions of Fellow in Physiology at Western Reserve University School of Medicine, Cleveland, OH; Associate Professor of Physiology at Bowman-Gray School of Medicine, Wake Forest University Winston-Salem, NC; Established Investigator of the American Heart Association; Visiting Associate in Engineering, California Institute of Technology, Pasadena; Fulbright Lecturer/Researcher, Montevideo, Uruguay; and the first Director of the Virginia Mason Research Center, Seattle, WA. He co-invented the square wave electromagnetic flowmeter and the DopScan imaging system. He founded and is currently the Executive Director of the Institute of Applied Physiology and Medicine, and is also Medical Director at Spencer Technologies. Among his research and clinical interests is the investigation of cardiovascular and cerebrovascular physiology using ultrasound.

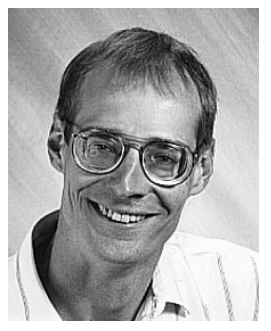

Douglas L. Jones received the B.S.E.E., M.S.E.E., and Ph.D. degrees from Rice University, Houston, TX, in 1983, 1986, and 1987, respectively. During the 1987-1988 academic year, he was at the University of Erlangen-Nuremberg in Germany on a Fulbright postdoctoral fellowship.

Since 1988, he has been with the University of Illinois at Urbana-Champaign, where he is currently a Professor in Electrical and Computer Engineering, the Coordinated Science Laboratory, and the Beckman Institute. In Spring 1995, he was on sabbatical at the University of Washington, Seattle. From July through September 1998, he was a Participant in the Programme on Nonlinear and Nonstationary Signal Processing at the Isaac Newton Institute for Mathematical Sciences at the University of Cambridge, Cambridge, U.K. In the Spring semester of 1999, he served as the Texas Instruments Visiting Professor at Rice University, Houston, TX. He is an author of the laboratory textbook A Digital Signal Processing Laboratory Using the TMS32010. His research interests are in digital signal processing, including time-frequency and time-varying signal analysis, communications systems, and various applications.

Dr. Jones is currently serving as an Associate Editor for the IEEE SIGNAL PROCESSING LeTters. 